\title{
Effects of exteroceptive feedback and safe-box confinement durations on escape behavior in rats
}

\author{
J. J. FRANCHINA, J. S. KASH, J. R. REEDER, and C. T. SHEETS \\ Virginia Polytechnic Institute and State University, Blacksburg, Virginia 24061
}

\begin{abstract}
In Experiment 1, rats received escape training in which an exteroceptive feedback cue occurred in the safe box, and safe-box confinement durations of 5 or $20 \mathrm{sec}$ were combined orthogonally with shock-box confinement durations of 5 or 20 sec. Exteroceptive feedback reliably facilitated escape performance relative to no-feedback controls when safe-box confinement was relatively longer than shock-box confinement. Confinement duration also facilitated performance in the absence of exteroceptive feedback. In Experiment 2, escape training with or without feedback was followed by extinction either with the feedback condition of prior training or with the opposite feedback condition. Feedback presentations in extinction reliably increased responding. Feedback removal reliably decreased responding relative to feedback controls. Introduction of feedback in extinction reliably enhanced performance relative to no-feedback controls.
\end{abstract}

Acquisition of escape (Tarpy \& Koster, 1970; Tarpy \& Sawabini, 1975) and avoidance behavior (Bolles \& Grossen, 1970; D’Amato, Fazzaro, \& Etkin, 1968; Feldman, 1977; Keehn \& Nakkash, 1959) are enhanced by the response-contingent presentation of a distinctive external cue in the safe situation. Bolles and Grossen (1970) and Tarpy and Koster (1970) have proposed that such external cue presentations following escape/avoidance behavior provide feedback which indicates the termination of the aversive situation and marks the start of a relatively safe, shock-free, period.

Bolles and Grossen (1970) also proposed that the effectiveness of exteroceptive feedback may be related to the duration of the safe period which follows responding. According to Denny (1971), the duration of confinement in the safe box following escape/avoidance performance provides for the occurrance of relief/relaxation behaviors. Relief is a relatively short-lived response with an onset at about $5 \mathrm{sec}$ postshock and a peak 10-15 sec later. Relaxation behavior starts $25-40 \mathrm{sec}$ after aversive stimulus termination and reaches a maximum at about $150 \mathrm{sec}$. Relief/relaxation responses are presumed to establish positive properties to stimuli in the safe box and, consequently, may serve to mediate approach towards the safe situation. If the role of exteroceptive feedback functions as Bolles and Grossen (1970) and Tarpy and Koster (1970) suggest, then safe-

These data were reported at the Midwestern Psychological Association meeting in Chicago, May 1975. Thanks are due to W. B. Pavlik for his thoughtful comments about this manuscript. Requests for reprints should be sent to J. J. Franchina, Department of Psychology, Virginia Polytechnic Institute and State University, Blacksburg, Virginia 24061. box confinement durations which foster occurrences of relief/relaxation behaviors should enchance the effects of feedback on escape/avoidance performance.

Franchina and Schindele (1975) reported that escape behavior followed by $20 \mathrm{sec}$ of safe-box confinement was reliably better than that followed by $5 \mathrm{sec}$ of confinement. These temporal intervals presumably fostered the occurrence of relief behavior in the safe situation (Denny, 1971). If safe-box conditions which promote relief/relaxation regulate the effectiveness of exteroceptive feedback on instrumental performance, then the results of Franchina and Schindele (1975) suggest that (relative to nofeedback controls) feedback effects should be better for long $(20 \mathrm{sec})$ as compared to short $(5 \mathrm{sec})$ confinement periods. The first experiment in this report evaluated this possibility for escape behavior. Bolles and Grossen (1970) have reported that feedback facilitated shuttle-avoidance behavior followed by a safe period $90 \mathrm{sec}$ long but not by one $30 \mathrm{sec}$ long. Denny (1971) has proposed that a 90-sec safe period fosters the occurrence of relaxation behavior.

\section{EXPERIMENT 1}

\footnotetext{
Method

Apparatus. The apparatus was described by Franchina, Bush, Kash, Troen, and Young (1973, Experiment 3). Briefly, a shock box was separated from a safe box by a guillotine door and a hurdle. The inside walls of each box were covered with Plexiglas inserts, $.64 \mathrm{~cm}$ thick, painted glossy white. The floor surface was constructed of stainless steel rods. The illumination level in each box was $110 \mathrm{fc}$. The exteroceptive feedback stimulus (FS) was a $1,200-\mathrm{Hz}$ tone from an audio generator (General Motors Corp.), and was delivered through a Fisher loudspeaker $(10 \mathrm{~cm}$ in diameter). The midline of the speaker was $10 \mathrm{~cm}$ behind the midpoint of the side wall of the safe box. Onset of the FS
} 
increased the ambient noise level from 52 to $74 \mathrm{~dB}$, as measured at the floor of the safe box.

Subjects. Eighty experimentally naive, female, hooded rats (Long-Evans descendants) were obtained from the local departmental colony. The rats were $100-120$ days old at the start of the experiment. Throughout the study, they were housed in pairs with food and water available ad lib.

Design and Procedures. On Day 1, each rat received $6 \mathrm{~min}$ of apparatus exploration. On Days 2 through 7 , the rats all received 10 escape training trials per day with presentation of FS in the safe box following escape performance ( $n=40$ rats) or with no explicit FS ( $n=40$ rats). For half of the rats in FS and in no-FS $(\overrightarrow{\mathrm{FS}})$ conditions $(\mathrm{n}=20 \mathrm{each})$ the safe-box confinement duration was $5 \mathrm{sec}$; for the other haif of the rats in FS and in $\overline{F S}$ conditions, confinement duration was $20 \mathrm{sec}$.

Franchina and Schindele (1975) and Weisman, Denny, and Zerbolio (1967) have suggested that the effects of safe-box confinement duration on performance may not relate to the absolute length of the confinement period but rather may relate to the relative durations of confinement existing between safe and shock boxes. To accommodate this possibility, two durations of shockbox confinement, 5 and $20 \mathrm{sec}$ preshock, were orthogonally combined with each of the safebox confinement durations. The resulting combinations of confinement and feedback variables yielded a $2 \times 2 \times 2$ factorial design: two feedback conditions (FS and $\overline{F S})$, two safe-box confinement durations ( 5 and $20 \mathrm{sec}$ ), and two shock-box confinement durations ( 5 and $20 \mathrm{sec}$ ).

For daily escape training procedures, the rats were brought into a darkened experimental room in pairs, one rat from FS and the other from $\overline{\mathrm{FS}}$, but daily training (10 trials) was completed for one rat before it started for the other. During the training of one rat, its partner remained in one of two unpainted holding boxes, each $24 \times 10 \times 6 \mathrm{~cm}$ and covered with a wire-mesh top. The boxes were approximately $60 \mathrm{~cm}$ away from the apparatus. Escape trials followed procedures from Franchina et al. (1973). Each rat was placed into the shock box, and after the appropriate 5 - or $20-\mathrm{sec}$ interval the guillotine door opened, thereby activating a $1.0-\mathrm{mA}$ shock and a timer calibrated in $.01-\mathrm{sec}$ units. If the rat jumped the hurdle within $40 \mathrm{sec}$, the rat's weight depressed the safe-box floor, the latency timer stopped, and the FS sounded (or no FS occurred) for the duration of confinement. After 5 or $20 \mathrm{sec}$ of confinement in the safe box under the appropriate feedback conditions, the rat was removed to a holding box until the next trial. Definition of the ITI in this study followed that proposed by Denny and Weisman (1964). The ITI was held constant at $30 \mathrm{sec}$ for each rat, but it consisted of the duration of confinement in the safe box $(5$ or $20 \mathrm{sec})$ plus a complementary period of confinement, 25 or $10 \mathrm{sec}$, respectively, in the holding box. If the rat failed to jump the hurdle within $40 \mathrm{sec}$ after shock onset, shock remained on and the rat was removed from the shock box to the holding box. In these cases, the latency recorded was $40 \mathrm{sec}$. Within each pair of rats, the order of running was alternated daily. The measure of performance, latency of hurdle-jumping, was converted into reciprocal scores.

\section{Results}

Figure 1 shows that feedback and confinement variables yielded negligible effects on performance in early training (Trial Blocks 1 and 2). However, on Trial Blocks 4 through 6 , the $5 / 20$ confinement groups performed better than any other group; moreover, feedback presentations substantially enhanced performance in the $5 / 20$ condition. Analysis of variance over all the data of Figure 1 yielded a reliable quadruple interaction, Feedback by Safe-box Confinement by Shock-box Confinement by Trial Blocks $[F(5,360)=3.57, p<.01]$. Analysis of variance of the data on Trial Blocks 1 and 2 revealed no reliable effects of feedback or confinement variables. Analysis of variance of the data over Trial Blocks 5 and 6 yielded a reliable interaction of Feedback by Safe-box Confinement by Shockbox Confinement $[F(1,72)=4.76, p<.025]$. The factor, Trial Blocks, was not reliable in the last analysis, nor did it enter into any reliable interactions. Paired comparisons (Scheffé tests) between confinement groups in FS and in FS conditions separately showed that Group 5/20 differed reliably from all other confinement groups (ps $<.05$ at least) in each condition. No other differences due to confinement duration were reliable. Comparisons between FS and $\overline{\mathrm{FS}}$ for each confinement level showed a reliable FS- $\overline{\text { FS }}$ difference only in the case of Group $5 / 20$ $[F(1,18)=5.74, p<.05]$.

\section{Discussion}

Experiment 1 showed that exteroceptive feedback

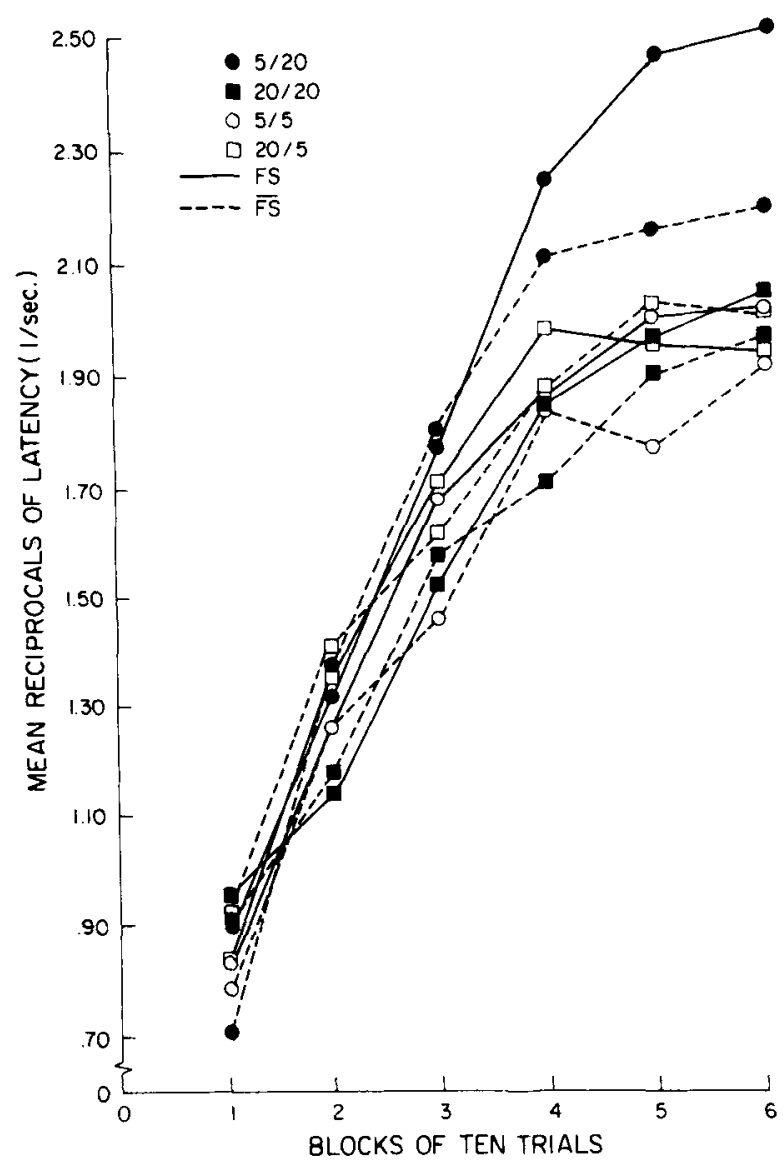

Figure 1. Mean reciprocals of latency of hurdle jumping in blocks of 10 training trials for feedback (FS) and no feedback (FS) conditions, with either 5 or 20 sec of shock-box confinement and 5 or 20 sec of safe-box confinement. Legend indicates "shock-box/ safe-box" confinement durations. 
facilitated escape performance when safe-box confinement duration was relatively longer $(20 \mathrm{sec})$ than shock-box confinement $(5 \mathrm{sec})$. The finding that feedback effects depended on relative confinement duration does not support Bolles and Grossen's (1970, p. 168) proposal that the effectiveness of feedback is largely dependent on the length of the shock-free (safe) period. In the present study, FS Group 20/20 received the same duration of safe-box confinement as did FS group 5/20 and each of these groups received a longer confinement period in the safe box than did FS Group 5/5. Nevertheless, the results showed that escape performance was reliably better for FS Group 5/20 (relative to its FS control) than for FS Groups $20 / 20$ and 5/5, which did not differ reliably from their $\overline{\mathrm{FS}}$ controls or from each other.

It seems unlikely that the difference in performance between Group 5/20 and Groups 5/5 and 20/20 was simply due to the distinctiveness which accrued to shock and safe boxes because of the temporal discrepancy between them in Group 5/20 and not in Groups 5/5 and 20/20 (Franchina \& Schindele, 1975). Group 20/5 had the same discrepancy in confinement durations between shock $(20 \mathrm{sec})$ and safe $(5 \mathrm{sec})$ boxes as Group 5/20 did; yet Group 5/20 reliably outperformed Group 20/5, and the latter showed no reliable effects of feedback stimulation. Thus, the present data strongly suggest that the relative length of the shock-free (safe) period may be the influential factor in demonstrating feedback effects.

\section{EXPERIMENT 2}

Experiment 1 showed that, under appropriate confinement conditions, presentation of exteroceptive feedback in the safe box reliably facilitated escape training. Since feedback influenced the acquisition of escape performance, it should follow that, other things being equal, the continuation or removal of feedback should affect the maintenance of responding. Katzev and Henderson (1971, Experiment 2) reported extinction results consistent with this expectation. Removal of the feedback cue at the start of extinction reliably impaired responding relative to that of controls that received feedback throughout training and extinction. Unfortunately, their data also showed that extinction performance by continuous feedback controls did not differ reliably from that of a group which had never received feedback following responding. As a result, the data of Katzev and Henderson (1971) provide limited information about the effects of feedback on response maintenance. Removal of the feedback cue at the start of extinction in their study may have produced a change in the stimulus complex following re- sponding, and the resultant decline in extinction performance may have simply reflected stimulus generalization decrement.

Since several studies have reported that exteroceptive feedback facilitates response acquisition, the lack of compelling evidence for feedback effects on response maintenance raises questions about the generality of the role of feedback, and it might be construed that feedback effects are at best transient. The purpose of the present experiment was to study the effects of feedback presentations on the extinction of escape responding. In this experiment, shock- and safe-box confinement durations were constant at $10 \mathrm{sec}$ in order to attenuate the appearance of feedback effects in response acquisition and thus permit evaluation of extinction performance following comparable levels of training. Since $10-\mathrm{sec}$ confinement was beyond the duration which Denny (1971) proposed for the onset of relief behavior $(5 \mathrm{sec})$, relief should occur postshock and the positive properties which consequently accrue to the safe situation should be similar across groups. Thus, no acquisition differences might be expected. However, if the conditioning of relief to available stimuli in the safe box postshock were greater for feedback than for no-feedback groups [see McAllister \& McAllister (1965, 1971) for fear conditioning], then despite the absence of reliable acquisition effects of feedback, extinction performance might show feedback effects.

\section{Method}

The apparatus, subjects, and general procedures were the same as those described for Experiment 1 unless otherwise noted.

Design and Procedures. Forty experimentally naive female rats received 2 days of apparatus exploration ( $6 \mathrm{~min} /$ day), 6 days of excape training, and at least 4 days of extinction. In escape training, half of the rats $(n=20)$ received response-contingent exteroceptive feedback (tone) in the safe box (FS group) and the other half of the rats $(n=20)$ did not receive the feedback cue in the safe box ( $\overline{\mathrm{FS}}$ group). Escape-training procedures followed those of Experiment 1. The rats were brought into the darkened experimental room in pairs, one rat from $\mathrm{FS}$, the other from $\overline{\mathrm{FS}}$. One rat of the pair completed its 10 training trials before training started for the other rat, the second rat remaining in the holding box near the apparatus. When a rat in the FS group received the tone during its confinement $(10 \mathrm{sec})$ in the safe box, the other rat in the $\overline{F S}$ group was in the nearby holding box during tone presentations. (Tone onset increased the ambient noise level from 50 to $70 \mathrm{~dB}$ measured at the floor of the holding box.) Thus, exposures to the feedback cue were equal for FS and $\overline{\text { FS }}$ groups prior to the start of extinction. Within each pair of rats, the order of training was altered daily.

During extinction, half of the rats in each feedback condition of training $(n=10)$ received a continuation of that feedback condition in extinction (i.e., FS/FS and $\overline{\mathrm{FS}} / \overline{\mathrm{FS}}$ groups). The other half of the rats in each training condition of feedback $(n=10)$ received extinction with the opposite feedback condition from that of training (i.e., FS/ $\overline{\mathrm{FS}}$ and $\mathrm{FS} / \mathrm{FS}$ groups). In extinction, one rat at a time was brought into the experimental room and received 10 extinction trials. The procedures for training and extinction trials were the same as those described for training trials in Experiment 1, except for the difference in confinement duration in shock and safe boxes, $10 \mathrm{sec}$ in this study, and the omission 
of shock on extinction trials. The intertrial interval was the same for all rats and consisted of $10 \mathrm{sec}$ confinement in the safe box followed by 20 -sec confinement in the holding box before the next trial started. In addition to measuring latency of hurdle-jumping performance, this experiment also included a trials-to-criterion index of extinction. The extinction criterion was that the rat failed to jump the hurdle into the safe box within $10 \mathrm{sec}$ on four of five consecutive trials. However, all rats received a minimum of 4 days of extinction, 10 trials per day, without regard to the extinction criterion.

\section{Results}

Figure 2 presents mean reciprocals of latency of hurdle jumping on the last two trial blocks of training and over Trial Blocks $1-4$ of extinction. At the end of training, performance of the FS groups was slightly superior to that of the $\overline{\mathrm{FS}}$ groups. However, analysis of variance over all the acquisition data and over the last two trial blocks of acquisition showed no reliable differences between feedback conditions $[F(1,36)=3.54$ and 2.20 , respectively, $\mathrm{p}>.05]$.

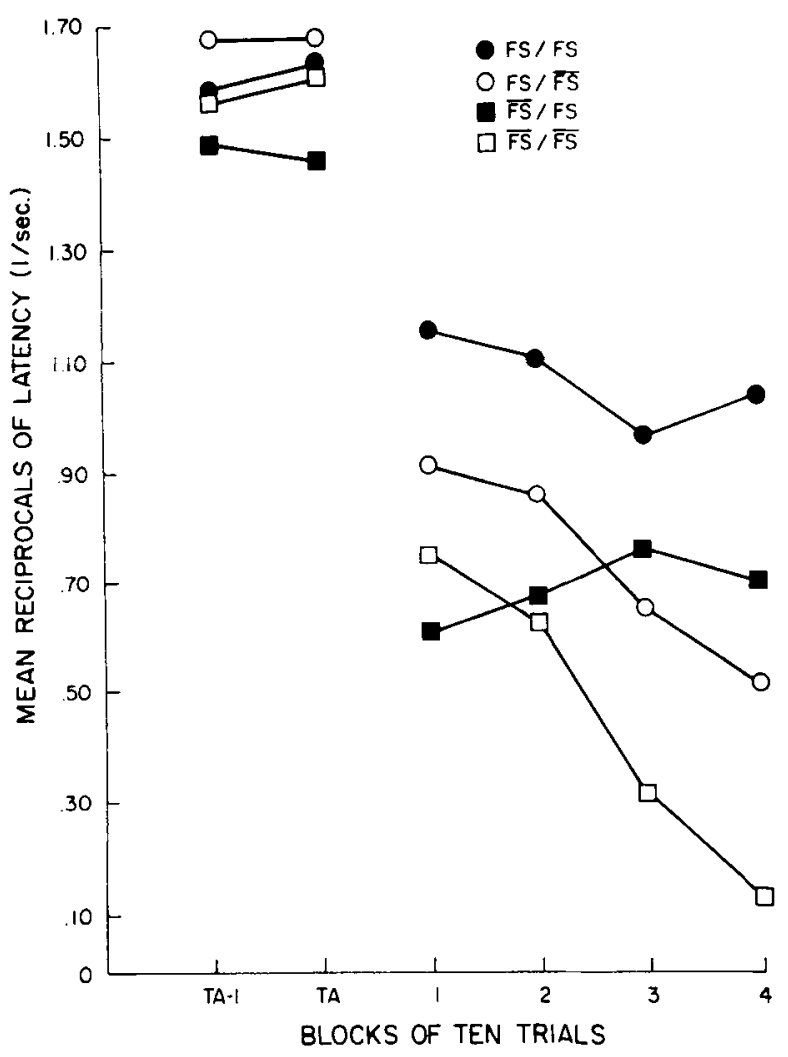

Figure 2. Mean reciprocals of latency of hurdle jumping in blocks of 10 trials for the last two blocks of training and for four blocks of extinction for groups which received exteroceptive feedback (FS/FS) or no feedback ( $\overline{\mathrm{FS}} / \overline{\mathrm{FS}}$ ) throughout training and extinction and for groups which were shifted from feedback in training to no feedback in extinction (FS $/ \overline{\mathrm{FS}})$, and vice versa

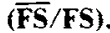

Figure 2 shows that during extinction Group FS/ FS, which received the feedback cue in training and extinction, showed the best performance and Group $\overline{\mathrm{FS}} / \overline{\mathrm{FS}}$, which never received responsecontingent feedback, showed the poorest performance. Removal of the feedback cue at the start of extinction (Group FS/ $\overline{\mathrm{FS}}$ ) decreased performance below the level of Group FS/FS but not to the level of Group $\overline{\mathrm{FS}} / \overline{\mathrm{FS}}$. Conversely, introduction of feedback stimulation at the start of extinction (Group $\overline{\mathrm{FS}}$ / FS) sustained performance at a level above that of Group $\overline{\mathrm{FS}} / \overline{\mathrm{FS}}$; but it did not improve performance to the level of Group FS/FS.

Analysis of variance over all the data of Trial Blocks 1-4 yielded reliable effects for Acquisition Feedback Condition $[\mathrm{F}(1,36)=6.27, \mathrm{p}<.025]$, Extinction Feedback Condition $[\mathrm{F}(1,36)=4.62, \mathrm{p}<.05]$, and Trial Blocks $[\mathrm{F}(3,108)=8.83, \mathrm{p}<.005$, and reliable interactions for Trial Blocks by Extinction Feedback Condition and Trial Blocks by Acquisition Feedback Condition by Extinction Feedback Condition $[\mathrm{F}(3,108)$ $=9.17$ and $2.83, \mathrm{p}<.005$ and $<.05$, respectively $]$. Subsequent analysis of variance explained the reliable interactions: namely, in the comparison of Groups FS/ FS and $\overline{\mathrm{FS}} / \mathrm{FS}$, the Groups effect was reliable $[F(1,18)=4.70, p<.05]$ but the Trial Blocks effect and the Groups by Trial Blocks interaction were not; in the comparison between Groups FS/ $\overline{\mathrm{FS}}$ and $\overline{\mathrm{FS}}$ / $\overline{\mathrm{FS}}$, the Trial Blocks effect was reliable $[\mathrm{F}(3,54)=$ $20.13, p<.001]$ but the Groups effect and the interaction were not.

For the criterial index of extinction, mean trials to criterion were 93.4 for Group FS/FS, 65.1 for Group $\overline{\mathrm{FS}} / \mathrm{FS}, 60.0$ for Group FS/FS, and 32.3 for Group $\overline{\mathrm{FS}} / \overline{\mathrm{FS}}$. The results of Groups FS/FS and $\overline{\mathrm{FS}} / \mathrm{FS}$ indicated that resistance to extinction was improved whether feedback presentations occurred in extinction or in training. Comparison of the mean trials to criterion for the $\overline{\mathrm{FS}} / \mathrm{FS}$ and $\mathrm{FS} / \overline{\mathrm{FS}}$ groups combined (62.5 trials) with means for the FS/FS and $\overline{\mathrm{FS}} / \overline{\mathrm{FS}}$ groups showed that the effects of presenting feedback in either session summated rather directly. That is, Groups FS $/ \overline{\mathrm{FS}}$ and $\overline{\mathrm{FS}} / \mathrm{FS}$ combined were superior to Group $\overline{\mathrm{FS}} / \overline{\mathrm{FS}}$ by roughly the same amount $(62.5-32.3=30.2$ trials $)$ as the combined groups were inferior to Group FS/FS $(93.4-62.5=30.9$ trials $)$. Analysis of variance over the trials-to-criterion data yielded reliable effects for Acquisition Feedback Condition and for Extinction Feedback Condition $[\mathrm{F}(1,36)=4.78$ and $6.69, \mathrm{p}<.05$ and $<.001$ ]. The Acquisition Feedback Condition by Extinction Feedback Condition interaction was not reliable $(F<1)$. Scheffé tests indicated that there were reliable group differences within each paired group comparison except for the comparison of Group FS/ $\overline{F S}$ with Group $\overline{F S} / F S$. 


\section{Discussion}

In Experiment 2, continuation or removal of exteroceptive feedback following training reliably affected extinction of escape responding. For example, Group FS/FS showed reliably greater resistance to extinction than did Group $\overline{\mathrm{FS}} / \overline{\mathrm{FS}}$. Removal of the feedback cue at the start of extinction (Group FS $/ \overline{\mathrm{FS}})$ reliably decreased performance relative to controls which received uninterrupted feedback presentations (Group FS/FS). The difference in extinction between Groups FS/FS and $\overline{\mathrm{FS}} / \overline{\mathrm{FS}}$ was especially important because it suggested that the response decrement for Group FS/FS was the result of the removal of a response-maintaining stimulus (the feedback cue) and not simply the disruptive consequence of stimulus change. Unfortunately, the extent of this decrement was ambiguous, because GroupFS/FS differed reliably from no-feedback controls on the trials-to-criterion index of extinction but not on the speed index; and present procedures did not permit a compelling basis for choosing between these results.

The findings of reliable feedback effects in extinction but not in training may seem puzzling, but as indicated earlier these data are not inexplicable from Denny's viewpoint. Ten seconds' confinement in the safe box during training in this study exceeded the duration which Denny (1971) proposed for the postshock onset of relief behavior. Consequently, relief may have occurred similarly across groups in training and relief's positive properties may have mediated comparable levels of approach for all groups. Thus no reliable group differences occurred in training.

During training, however, postshock relief may have become conditioned to safe-box stimuli, conditioning being stronger to the stimulus compound which consisted of the discrete feedback cue and static cues of the safe box in the FS condition than to static cues alone in the $\overline{\mathrm{FS}}$ condition (see McAllister \& McAllister, 1971). Extinction under nonshock conditions eliminated postshock relief from the safe situation. However, safe-box stimuli presumably elicited conditioned relief following responding and relief's positive properties served to maintain performance toward the safe situation. Accordingly, Group FS/FS performed better than Group $\overline{\mathrm{FS}} / \overline{\mathrm{FS}}$, because feedback presentations elicited conditioned relief more strongly for GroupFS/FS and thereby sustained responding over that for Group $\overline{\mathrm{FS}} / \overline{\mathrm{FS}}$. It was also possible that extinction for Group $\overline{\mathrm{FS}} / \overline{\mathrm{FS}}$ reflected the backchaining of conditioned relief (Denny, 1971) from the safe box to highly similar cues in the shock box. Denny, Koons, and Mason (1959) and Franchina et al. (1973) reported that extinction performance was reliably poorer when shock and safe boxes were similar rather than dissimilar to each other. They ascribed their results to the backchaining of relief/ relaxation. In the present study, backchaining may have fostered the occurrence of conditioned relief in the shock box during extinction for Group $\overline{\mathrm{FS}} /$ $\overline{\mathrm{FS}}$. Relief may have interfered with the aversivemotivational basis (fear) of escape behavior, thereby impairing escape performance and facilitating extinction.

For Group FS/ $\overline{\mathrm{FS}}$, the level of conditioned relief established in training should have been comparable to that of Group FS/FS, since both groups were exposed to the compound stimulus (tone and static cues) in the safe box. However, removal of the feedback cue for Group FS/ $\overline{\text { FS }}$ at the start of extinction may have reduced the amount of conditioned relief available to sustain responding toward the safe situation and performance dropped below the level of Group FS/FS.

The present experiment also showed that the introduction of the feedback stimulus into the safe box at the start of extinction reliably retarded extinction relative to no-feedback controls. Since the feedback cue did not accompany postshock relief in the safebox during training for Groups $\overline{\mathrm{FS}} / \mathrm{FS}$ or $\overline{\mathrm{FS}} / \overline{\mathrm{FS}}$, their respective levels of conditioned relief should have been comparable to each other at the start of extinction. However, for Group $\overline{\mathrm{FS}} / \overline{\mathrm{FS}}$, the backchaining of conditioned relief to shock-box cues presumably facilitated extinction, while for Group $\overline{\text { FS}} / \mathrm{FS}$ the introduction of the exteroceptive feedback cue in the safe box may have made the safe situation sufficiently discriminable from the shock box to attenuate the extent of backchaining. Thus, the difference in extinction performance between Groups $\overline{\mathrm{FS}} / \mathrm{FS}$ and $\overline{\mathrm{FS}} / \overline{\mathrm{FS}}$ may not have reflected the enhancing effects of feedback and conditioned relief on Group $\overline{F S} / F S$ 's performance but rather the relatively more rapid backchaining of relief to the shock box for no-feedback controls than for Group $\overline{\text { FS } / F S . ~}$

\section{GENERAL DISCUSSION}

The results of Experiments 1 and 2 together are more consistent with Denny's (1971) relief/relaxation theory than with Bolles and Grossen's (1970) feedback hypothesis. For example, across the two experiments, the acquisition data failed to yield reliable feedback effects for shock- and safe-box conditions, $5 / 5,10 / 10$, and 20/20. Bolles and Grossen (1970) proposed that the effectiveness of feedback critically depended on safe-box confinement durations which fostered relief/relaxation behaviors. Thus, feedback effects should have increased directly for the $5 / 5$, $10 / 10$, and $20 / 20$ conditions because these intervals encompassed the durations which presumably occasioned postshock relief behavior. In these experiments, feedback yielded reliable effects only in 
the relational $(5 / 20)$ condition.

The extinction data of Experiment 2 are consistent with the feedback viewpoint in that responding was related to the continuation or removal of feedback during extinction. However, it is uncertain how the feedback hypothesis would explain the finding of reliable feedback effects in extinction following nondifferential feedback effects in acquisition or the finding of reliable feedback effects for Group $\overline{\mathrm{FS}} /$ FS, which received the feedback cue only during extinction. These data seem puzzling because the feedback hypothesis proposes that informational value accrues to a feedback cue during training as a result of the cue's indicating the start of a shockfree period. Admittedly the current status of the feedback hypothesis is not that of a formalized proposition; and it may be unreasonable to demand from it a more specific analysis than the hypothesis can currently provide. However, it should be noted that the relief/relaxation theory can explain more of the present data more easily and with fewer qualifications than can a feedback explanation.

\section{REFERENCES}

Bolles, R. C., \& Grossen, N. E. Function of the CS in shuttlebox avoidance learning by rats. Journal of Comparative and Physiological Psychology, 1970, 70, 165-169.

D'Amato, M. R., Fazzaro, J., \& Etkin, M. Anticipatory responding and avoidance discrimination as factors in avoidance conditioning. Journal of Experimental Psychology, 1968, $77,41.47$.

DenNy, M. R. Relaxation theory and experiments. In F. R. Brush (Ed.), Aversive conditioning and learning. New York: Academic Press, 1971.

Denny, M. R., Koons, P. B., \& Mason, J. E. Extinction of avoidance as a function of the escape situation. Journal of Comparative and Physiological Psychology, 1959, 52, 212-214.
Denny, M. R., \& Weisman, R. G. Avoidance behavior as a function of length of nonshock confinement. Journal of Comparative and Physiological Psychology, 1964, 58, 252-257.

Feldman, M. A. The effects of preexposure to a safety signal on the acquisition of a two-way avoidance response in rats. Animal Learning \& Behavior, 1977, 5, 21.24.

Franchina, J. J., Bush, M. E., Kash, J. S., Troen, D. M., \& Young, R. L. Similarity between shock and safe areas during acquisition, transfer, and extinction of escape behavior in rats. Journal of Comparative and Physiological Psychology, 1973, 84, 216-224.

Franchina, J. J., \& Schindele, T. E. Nonshock confinement duration and shock- and safe-box similarity during escape training in rats. Animal Learning \& Behavior, 1975, 3, 297-300.

Katzev, R. D., \& Henderson, R. W. Effects of exteroceptive feedback stimuli on extinguishing avoidance responses in Fischer344 rats. Journal of Comparative and Physiological Psychology, 1971, 74, 66-74.

KeEhN, J. D., \& NakKash, S. Effect of a signal contingent upon an avoidance response. Nature, 1959, 184, 566-568.

MCAllister, W. R., \& McAllister, D. E. Variables influencing the conditioning and the measurement of acquired fear. In W. F. Prokasy (Ed.), Classical conditioning: A symposium. New York: Appleton, 1965

MCAllister, W. R., \& MCAllister, D. E. Behavioral measurement of conditioned fear. In F. R. Brush (Ed.) Aversive conditioning and learming. New York: Academic Press, 1971.

TARPY, R. M., \& Koster, E. D. Stimulus facilitation of delayedreward learning in the rat. Journal of Comparative and Physiological Psychology, 1970, 71, 147-151.

TARPY, R. M., \& SAWABINI, F. L. Stimulus facilitation of delayedreward performance as a function of the cue's spatial position. Bulletin of the Psychonomic Society, 1975, 6, 385-387.

Weisman, R. G., Denny, M. R., \& Zerbolio, D. J., JR. Discrimination based on differential nonshock confinement in a shuttle box. Journal of Comparative and Physiological Psychology, 1967, 63, 34-38.

(Received for publication June 24, 1977; revision accepted April 26, 1978.) 